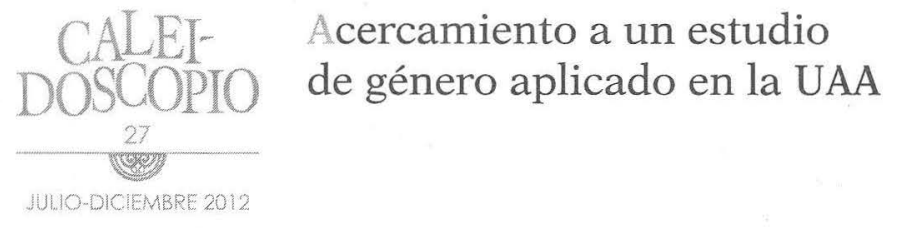

José Acevedo Acosta

Universidad Autónoma de Aguascalientes

INTRODUCCIÓN

En primer lugar, hay que decir que el diagnóstico de Equidad de Género en la UAA (Agencia Medium Publicidad/División Investigación, 2011), es un ejercicio de acercamiento y de opinión de la comunidad universitaria que fue bien recibido y despertó gran interés porque, siendo un tema de actualidad y con las repercusiones cívicas, sociales y culturales que ello implica, es necesario observar ese fenómeno mediante los instrumentos a nuestra disposición. Resultó un estudio más cuantitativo, con categorías conceptuales que es posible mejorar sobre la marcha, pues éstas ofrecen confusión o limitaciones de interpretación, al mismo tiempo que se percibe un acercamiento más externo que interno respecto de las preguntas que se aplicaron a la comunidad universitaria. Existe, por dar un solo ejemplo, la confusión entre género y sexo desde la mirada de perspectiva de género, que es una tarea por esclarecer.

Para el estudio que nos ocupa se utilizó una encuesta abierta a la población de la comunidad universitaria, en sus tres segmentos: estudiantes, docentes y personal administrativo. Dicho proceso representa un primer acercamiento institucional sobre algo que está en el interés nacional e internacional respecto a una vida social libre de prejuicios, de objeciones y efectos de convivencia en los espacios educativos y sociales de concurrencia regular y duradera. 
Cabe decir que el diagnóstico y sus resultados son incompletos, por ser la primera aproximación, porque todo intento llevado a cabo será perfectible y adaptable paulatinamente para otros universos y con otras miradas, con distintos sujetos y en condiciones diferentes. En siguientes ocasiones sería recomendable hacer una desagregación mayor, distinguiendo dentro de las categorías estudiantes, docentes y personal administrativo, la cuestión por sexo, edad, centros académicos o direcciones generales, entre otras categorías que puedan imaginarse como útiles y/o necesarias para los propósitos de investigaciones, de la universidad y de la entidad política nacional.

Lo que aquí nos proponemos es contemplar ese primer trabajo de diagnóstico que la UAA emprendió con el apoyo nacional del Programa Integral de Fortalecimiento Institucional (PIFI), acercarnos a las preguntas formuladas, percibir algunos de los resultados cuantitativos para realizar una interpretación sencilla de los mismos y difundirlos para que la comunidad universitaria los conozca. Del total de las preguntas hechas a cada segmento institucional únicamente observaremos con detalle dos de ellas y desde ahí haremos una extrapolación o interpretación de lo que nos pueden decir los resultados obtenidos por el instrumento aplicado.

Es importante advertir que el apartado de objetivo general y objetivos particulares fue tomado literalmente del diagnóstico (Estudio sobre la equidad de género en la UAA, 2011) para poder contrastar ese ejercicio de encuestas aplicado con la cavilación que aquí se ofrece.

\section{OBIETIVO GENERAL}

"Realizar un estudio con enfoque integral de género en donde a partir de los resultados que arroje dicho estudio, se promuevan proyectos de investigación entre las Direcciones de Educación Superior (DES) para la reivindicación de los derechos de mujeres y hombres" (Agencia Medium Publicidad/División Investigación, 2011).1

\section{Objetivos específicos}

- Conocer la opinión de la comunidad universitaria respecto a las prácticas a favor de los derechos de las mujeres.

1 Aquí se incluyen tanto el objetivo general como los objetivos particulares del estudio. 
- Conocer la opinión respecto a equidad entre hombres y mujeres que se da en la universidad.

- Observar la opinión respecto al nivel y/o formas de discriminación que se presentan en la institución.

- Conocer la opinión de la comunidad respecto las diferencias y similitudes que existen para cada género (hombre o mujer) en la generación de oportunidades laborales

- Detectar aquellas prácticas que puedan ser motivo de discriminación por aspectos de sexo o por políticas y procedimientos.

- Conocer la opinión de la comunidad universitaria, de si han sido víctimas de abuso sexual o represión en sus diversas expresiones por razones de diferencia de género hacia adentro de la institución.

Es una investigación de tipo descriptivo que permite medir de manera independiente los conceptos que se indagan, con un diseño no experimental, porque no existe el control de variables. La muestra se aplicó a 969 universitari@s ${ }^{2}$ en total: 270 personas de las 17 áreas administrativas, 326 docentes de los ocho Centros académicos y a 373 estudiantes de los diversos niveles de bachillerato, licenciatura y posgrado (Agencia Medium Publicidad/División Investigación, 2011)3.

\section{LOS NÚMEROS DEL DIAGNÓSTICO}

Para emprender el seguimiento del diagnóstico disponible, fechado el 15 de abril de 2011, podemos efectuarlo siguiendo una a una las preguntas dirigidas a cada sector, también podríamos iniciarlo fijándonos sólo en uno de los segmentos de la comunidad universitaria, o empezando por aquellas preguntas que se vuelven valiosos referentes por la relevancia o incidencia de aquellos datos que interesan para este propósito. Para este ensayo partiremos de dos preguntas que se formularon casi al final del cuestionario aplicado, tales interrogaciones se presentaron cuando prácticamentel@s participantes han recorrido

2 Es pertinente decir que se utilizan en dos o tres veces palabras con el signo de (@) para evitar repetir, por ejemplo las universitarias y los universitarios, lo hemos dejadoen l@sy universitari@s; se encontrarán otros usos semejantes.

3 Además, hay que señalar que el documento original menciona también a alumnos de secundaria, dato que preferimos omitir, pues el de educación básica de secundaria por parte de la UAA se dejó de impartir hace ya un par de años. 
todas las pistas o items que les pidió dicho instrumento, y pueden, entonces, los invitados interpretarlas para realizar una especie de cierre, evaluación, calificación o suma de sugerencias y recomendaciones de1@sentrevist@sydel ejercicio aplicado. Las preguntas son las mismas para las alumnas y los alumnos, las profesoras y los maestros y para los y las personas de las áreas administrativas, como lo veremos más adelante.

\section{Estudiantes}

El número total de preguntas para l@s estudiantes fue de 46, varias de ellas resultan irrelevantes para profundizar sobre el ejercicio, la visibilidad o la identificación de ciertos problemas sobre las relaciones de la comunidad escolar de la UAA, en torno a la equidad de género, de si hay preferencias, predisposiciones o menosprecio de las o los alumnos por razón de su sexo, en la convivencia entre pares de jóvenes, o en la relación natural que se establece entre docentes-estudiantes, o si acaso han observado que haya diferencias marcadas entre unas y otros. De esos 46 items, se entresacan únicamente aquellos que guardan mayor relevancia para nuestro propósito.

En total, son 19 preguntas las seleccionadas, por ser las más vinculadas con la investigación de campo aplicada en la UAA, porque pueden ser ideas o proposiciones que interesen a los lectores de este ensayo temático sobre equidad de género ${ }^{4}$. En cambio, se creyó innecesario incluir sus respuestas, pues ello llevaría a repetir, parcialmente, las conclusiones numéricas obtenidas por el diagnóstico institu cional, cuando lo que más se pretende es hacer algunas cavilaciones que nos conduzcan a profundizar más con las inquietudes que la comunidad universitaria expresó libremente.

1. ¿Considera que en la UAA se vive la igualdad de género?

2. ¿En el tiempo que tiene en la UAA, se ha sentido apartada(o) de algún evento por ser mujer/hombre/homosexual?

3. ¿Cree que existen carreras exclusivas para hombres y/o mujeres en la UAA? L@s universitarios respondieron. Para hom-

4 El texto completo del Diagnóstico multicitado aplicado en la UAA se encuentra en las oficinas de la Defensoría para su consulta. 
bres: Sistemas, Ingeniería Civil y Agronomía; para mujeres: Asesoría psicopedagógica, Trabajo Social, Pedagogía y Diseño de Modas. La enumeración mencionada puede parecer incompleta, es cosa de buscar un poco más acerca de ese carácter sexista que aún perdura entre profesiones.

4. ¿Sus compañeros(as) hacen comentarios ofensivos o burlescos hacia mujeres, hombres o hacia homosexuales?

5. ¿Sus compañeros(as) hacen comentarios ofensivos o burlescos hacia mujeres, hombres o hacia personas homosexuales?

6. ¿Sus maestros suelen hacer comentarios ofensivos hacia mujeres, hombres o hacia personas homosexuales?

7. ¿Sus maestras suelen hacer comentarios ofensivos hacia mujeres, hombres o hacia personas homosexuales?

8. ¿Ha escuchado al personal del área administrativa o de mantenimiento hacer comentarios ofensivos hacia mujeres, hombres o personas homosexuales?

9. ¿Los maestros le dan su lugar por igual a los alumnos hombres que a las alumnas mujeres?

10. ¿Las maestras le dan su lugar por igual a los alumnos hombres que a las alumnas mujeres?

11. ¿Le han dicho algún cumplido sobre su apariencia que le haya incomodado?

12. ¿Ha escuchado comentarios sobre su vida sexual que le incomoden?

13. ¿Ha sido víctima de contacto físico no deseado?

14. ¿Le han hecho invitaciones de tipo sexual?

15. ¿Le han chantajeado si no acepta alguna invitación fuera de la escuela?

16. ¿Se le ha forzado físicamente a tener contacto de tipo sexual?

17. ¿En términos generales, considera que existe trato desigual entre hombres y mujeres en la UAA?

18. ¿Cree necesario emprender acciones que fomenten la igualdad de oportunidades entre mujeres y hombres en la UAA? Se resaltan con negritas estas dos preguntas porque son las que servirán como referencia para el análisis que se propone desde aquí.

19. ¿Cuáles acciones consideras más adecuadas para fomentar la igualdad de oportunidades entre hombres y mujeres en la UAA? 
La primera cuestión de nuestro interés dice: ¿Cree que es necesario emprender acciones que fomenten la igualdad de oportunidades entre mujeres y hombres en la UAA?

Volviendo la mirada y la conciencia al ensayo analítico que se ofrece, descubrimos que $46.8 \%$ estima que sí es recomendable aplicar medidas particulares para fomentar la equidad de género, en tanto que $46.6 \%$ se inclina porque no hace falta. Dicha conclusión es tan pareja y equilibrada que a la universidad y a quienes atienden esos programas específicos no les dan herramientas de juicio suficientes para aplicar o no tales medidas, y, sin embargo, por otro lado, cualquiera de las dos respuestas resulta por ello mismo ilustrativa de las lagunas, ausencias y recomendaciones que la comunidad estudiantil descubre y plantea luego de esta breve experiencia. Es decir, aunque este segmento universitario expresó juicios más bien favorables de las relaciones de convivencia, también deja abierta la probabilidad y/o necesidad de prevenir, fomentar, educar, orientar o de impulsar acciones que eleven, no sólo el porciento de las percepciones, sino el comportamiento ordinario de la convivencia académica. Expresado en otros términos, ellas y ellos admiten que se viven ciertos valores morales aceptables, pero ciertamente perfectibles, y para ello hace falta un esfuerzo institucional adicional, que es la tarea que corresponderá a aquellos que están entreabriendo esta interesante parcela de la convivencia social, académica, humana y cultural entre la comunidad universitaria, vista con los ojos de las y los jóvenes de la UAA.

La siguiente pregunta formulada fue, partiendo de la respuesta favorable de que sí es recomendable emprender algunas acciones, ¿Cuáles acciones consideras más adecuadas para fomentar la igualdad de oportunidades entre hombres y mujeres en la UAA?. La población estudiantil emitió una serie de recomendaciones interesantes y viables para este propósito, siempre dando por hecho que es ampliamente recomendable mejorar esas relaciones humanas cotidianas. En orden descendente las propuestas fueron:

- Realizar una feria con conferencias, pláticas y foros informativos, ${ }^{5}$ que propicien la igualdad en el trato y relaciones estudiantiles universitarias. Tales actividades son ampliamente

5 Las recomendaciones hechas por estudiantes van señaladas con texto subrayado, lo que va después de esta indicación es un complemento de esa idea, de parte del autor de estas líneas. 
recomendables como insumos para transferir información, experiencias, ponderar áreas de oportunidad y modalidades aplicables para el objetivo específico; son acciones que pueden cruzarse o disponerse en tiempos oportunos o con calendarios anticipados. Regularmente son formatos impersonales y generales, pero que pueden representar acercamientos y momentos de análisis que cubran la expectativa de quienes estiman que son instrumentos válidos y convenientes en el corto plazo. ${ }^{6}$

- Tratarnos de igual manera. Es una recomendación callada pero firme y directa a la comunidad universitaria; tal vez, por tratarse de las opiniones de estudiantes, va más dirigida al personal docente en toda su gama, en sus diferentes espacios y modos de convivencia: áulica, laboratorios, talleres, seminarios, prácticas de campo, prácticas profesionales, servicio social, entre otras modalidades de convivencia y encuentro de ellas y ellos con las y los académic@s. ¿Tienen verdad l@s jóvenes en su propuesta para construir la equidad universitaria y cultural?

- Fomentar la participación equitativa. Lo están gritando claramente. Hay que promover intervenciones y ejercicios equitativos, respetuosos; además advierten de la recomendación de ser tomados en cuenta para llevar a cabo una participación más equitativa, igualitaria y profesional. El paso generacional se gesta en esos espacios comunes y se cultiva en el día a día, en el minuto a minuto, en las actitudes calladas y silenciosas pero siempre visibles y ejemplificantes, pues si se aplican adecuadamente dejan su estela favorable y se agradecen; cuando tales actitudes son deplorables, el rechazo comenzará en los salones, entre personaos, pero se extrapolarán en experiencias y vivencias que marcarán recuerdos que ell@s cuestionarán intermitentemente.

- Compartir actividades. La función de la docencia es una labor dialéctica e incluyente, recíprocamente, sea para bien o sea en

6 Es necesario informar que el Comité Institucional para la Equidad de Género (CIEG) ha ofrecido cursillos breves para estudiantes de la UAA, que han sido bien acogidos y que quienes los han tomado pidieron que se les impartieran otros más. Generalmente estas prácticas y mini cursos logran sacarlos de su vida ordinaria y los integran hacia una convivencia humana y universitaria. 
su sentido opuesto, ya que no existe el o la enseñante si no es para una aprendiz o un aprendiz, en espacios y tiempos determinados, en el aquí y el ahora como fenómeno humano, que al mismo tiempo es fenómeno educativo, social, cultural, político y ético, entre otros modos de valorarlo y/o de analizarlo.

- Responsabilidad igual en ambos sexos. Mirar a las y los estudiantes como iguales y diferentes, como personas con las mismas probabilidades y necesidades de preparación, disposición y compromiso de las acciones docentes y de los fenómenos concomitantes en ese proceso disciplinar de condiscípulos y docentes. Aprender juntos y compartir el ejercicio de estar y avanzar colegiadamente. El hecho de ser condiscípulos o de existir colegiadamente implica una conciencia mayor y una reiteración voluntaria frecuente para cultivar esa igual responsabilidad para ellas y ellos. Nos están señalando que más de una ocasión las relaciones de enseñanza-aprendizaje entre los sujetos del arte educativo no suceden así, que no todos, ni siempre, lo expresan explícitamente, y ello merece ser recordado de vez en cuando.

- Erradicar la creencia de actividades o carreras exclusivas de algún sexo. De unas décadas de años a la fecha la percepción y aceptación de existencia de carreras exclusivas para mujeres y hombres ha venido desvaneciéndose, porque la vivencia histórica muestra que las labores y faenas de la milicia, la navegación aérea y marítima, las ramas de la medicina humana y veterinaria, con sus respectivas especializaciones, así como la docencia en todos los niveles educativos, la enfermería, las ingenierías, las ciencias duras, la física, las bellas artes, las artes culinarias y, prácticamente, todas las tareas emprendidas por personas de ayer y de hoy han dejado atrás esa antigua tradición que separó, por centurias y milenios, la tácita exclusividad de campos de trabajo, de intervención y de poder social, científico, cultural, político y académico.

Hoy en día aquella división profesional y social de antaño se ha superado por la vía de los hechos. Para el caso de las instituciones de educación superior, es una obviedad que la matrícula estudiantil se ha equilibrado numéricamente, y se sabe que las preferencias profesionales han alterado la divi- 
sión educativa de antaño. Luego entonces, es prudente y recomendable que las y los profesores dejen de emitir juicios de valoración acerca de que aún existen parcelas profesionales propias y exclusivas para hombres o para mujeres, o evitar irritarse porque haya que aprehender muy íntimamente, en nuestro fuero personal docente, que ese sesgo sexuado o sexista de las carreras universitarias ya no existe más, y que la recomendación que hacen 1@s estudiantes es oportuna, adecuada y conducente. Cuánto tendrán que aprender las personas aplicadas a la docencia de las opiniones y cambios de mentalidades y culturales que ostentan y exigen est@s colegas de la universidad.

- Respeto entre compañeros. Dicha admonición no es de ahora, sino que ha de entenderse como una actitud permanente y generosa de parte de quienes ejercen el arte y profesión de la docencia, la investigación y la difusión en instituciones públicas y autónomas. Promover el respeto desde la cátedra es una exigencia moral, ética y profesional que mucho agradecerán, ahora, la juventud en proceso de formación, mañana, la sociedad entera, porque los principios y actitudes que se viven en el aula pasarán como legado cultural y moral de los egresados en el mundo laboral en pleno.

- Trabajo en equipos. Una oportunidad invaluable para homogeneizar información, habilidades y promover actitudes educativas es, justamente, la labor en equipos, pues en esos breves momentos y oportunidades en que se trabaja mediante pequeños grupos en el aula, se observan y perciben vivencias humanas de esa casi espontánea comunicación, sea entre pares de aula, de generación, con alumnos de la propia universidad o de otros centros escolares. El trabajo en equipo permite y aproxima en muchos sentidos a quienes participan en esa dinámica o actividad humana. Enseña a oír, escuchar, dialogar, interpelar, discutir y respetar y educarnos en el arte de construir colegiadamente lo que suele verse, con alguna frecuencia, como pérdida de tiempo. En cada área del conocimiento, en cada asignatura, esa tarea grupal tendrá distintas expresiones, y ello dependerá de otros elementos que deberán ser atendidos por la parte docente universitaria. 
Las ocho ideas advertidas por los estudiantes no representan novedades creativas o ingeniosas, simplemente están pidiendo una educación fincada en metodologías o estrategias didácticas que faciliten recrear esa anhelada igualdad, que lleven a establecer un trato igual entre iguales en las tareas aúlicas. No obstante, puede resultar novedoso que 1@s docentes se percaten de la expectativa que viven los jóvenes y de la ilusión que guardan, pero que están reclamando, obvio, porque regularmente no se les ofrece un enfoque discursivo y analítico en esas cosas sencillas de modo regular; tal vez porque ni las profesores y ni los profesoras saben o no intuyen eso que ellos esperan. Por esto resulta tan valioso que esta incipiente cultura de intercomunicación y diálogo recíproco, a propósito del diagnóstico sobre equidad de género, se comparta a todos los sectores universitarios. La generosidad de la juventud es muy clara y comparten nítidamente sus iniciativas como propuestas viables que 1@s docentes deberán asumir como una forma de comunicación necesaria y permanente.

Sin lugar a dudas, las apreciaciones estudiantiles pueden ser el reflejo de sus años de convivencia con personal docente y administrativo, y es desde la experiencia que ellos observan y plantean sus juicios, para que sean atendidos, adecuadamente, por el resto de la comunidad universitaria.

\section{Personal administrativo}

Las preguntas formuladas para este segmento fueron 54 , y se seleccionaron 25 como las más relevantes para este seguimiento.

1. ¿Considera que en la UAA se vive la igualdad de género?

2. ¿Le han negado algún permiso en su trabajo por ser mujer/ hombre /homosexual?

3. ¿Le han hecho sentir mal por ser mujer/hombre/homosexual en su trabajo?

4. ¿Considera que se le ha negado el ascenso o promoción a un puesto superior por el simple hecho de ser hombre/ mujer / homosexual?

5. ¿Hay otras personas en su área que por ser de diferente sexo ya se les haya otorgado algún ascenso? 
6. ¿En el tiempo que tiene en la UAA, se ha sentido apartada(o) de algún evento por ser mujer/hombre /homosexual?

7. ¿Considera que haya algún puesto exclusivo para hombres y/o mujeres en la UAA? Para hombres: vigilancia, mantenimiento, manejo de máquinas, rector, supervisor, ordeña, velador, jefatura, ingeniero en sistemas, decano, almacén. Propios para mujeres: intendencia y área secretarial.

8. ¿Recibió un trato desigual en las capacitaciones por parte del instructor por ser hombre/mujer/homosexual?

9. ¿Cree que el no tener un salario justo se deba por alguna situación de discriminación por diferencia de sexos?

10. ¿Se siente satisfecho(a) con el puesto que tiene actualmente?

11. En términos generales, ¿considera que existe trato desigual entre hombres y mujeres en la UAA?

12. ¿Sus compañeros hacen comentarios ofensivos o burlescos hacia mujeres, hombres o personas homosexuales?

13. ¿Sus compañeras hacen comentarios ofensivos o burlescos hacia hombres, mujeres o personas homosexuales?

14. ¿Sus superiores suelen hacer comentarios ofensivos hacia mujeres, hombres o personas homosexuales?

15. ¿Sus superiores le dan su lugar por igual a los hombres y a las mujeres de su área?

16. ¿La mujer tiene que esforzarse más que el hombre para progresar en su trabajo?

17. ¿Le han dicho algún cumplido sobre su apariencia que se haya incomodado?

18. ¿Ha escuchado comentarios sobre su vida sexual que le incomoden?

19. ¿Ha sido víctima de contacto físico no deseado?

20. ¿Considera que el ambiente que le rodeas es sexista (privilegiar un género por encima del otro)?

21. ¿Le han hecho invitaciones de tipo sexual?

22. ¿Le han chantajeado si no acepta alguna invitación fuera de la Universidad?

23. ¿Se le ha forzado físicamente a tener contacto de tipo sexual?

24. ¿Cree necesario emprender acciones que fomenten la igualdad de oportunidades entre mujeres y hombres en la UAA? 


\section{5. ¿Cuáles acciones considera más adecuadas para fomen- tar la igualdad de oportunidades entre hombres y mujeres en la UAA?}

¿Cuáles han sido las respuestas vertidas por los entrevistados del personal administrativo, respecto a esta misma cuestión? Lo que se expone a continuación sirve para resaltar su propia percepción y para comparar lo que éstos viven y esperan de la universidad y lo que planteó la juventud estudiosa en 4.1. Vayamos a los resultados.

$¿ C$ Cree necesario emprender acciones que fomenten la igualdad de oportunidades entre mujeres y hombres en la UAA?

Para este caso, los números son ilustrativos porque resaltan una serie de ideas que deben valorarse en su justa dimensión. Recuérdese que la respuesta de estudiantes fue casi igual entre el sí y el no (46\%) que hace falta un programa de acciones en este sentido. En cambio 1@s encuestados del personal administrativo responden que sí es necesario ofrecer acciones que fomenten la igualdad de oportunidades entre mujeres y hombres, con 169 respuestas que representan el 63\%, dato que se complementa con las 83 que asientan que no es menester (30.7\%) y 17 más que no emitieron su opinión.

Si este concentrado se incrementa exponencialmente, tomando en cuenta el número total de personas del área administrativa, resulta preocupante lo que estos números arrojan, ya que el 63\% representa que dos de cada tres trabajadores perciben irregularidades, intuyen preferencias o sienten en su vida y en su persona una desigualdad laboral dentro de la UAA, pues si la convivencia laboral funcionara con mayor equidad, seguramente el porcentaje no fuese tan elevado ni tan alarmante. Sin duda la tarea para este sector es la más urgente, porque denota desigual y demanda atención institucional de modo inmediato, en consecuencia, con más urgencia una atención profesional. La percepción del contingente administrativo debe valorarse con otras mediciones, porque son ell@s los que están en todas las áreas públicas, ventanillas, pasillos, cubículos, laboratorios, campos experimentales, en áreas de apoyo a la administración central, Rectoría, direcciones, decanatos, jefaturas académicas y administrativas. Ell@s son la vía de acceso e intercambio entre la sociedad hidrocálida, empresarial, familiar, educativa, juvenil, vocacional y demás, dado que 
para acceder a las áreas de ejecución, dirección y de representación universitaria, la mediación y la bienvenida la efectúan esas más de mil personas que identificamos con el término genérico de personal administrativo. ¿Qué razones habrá detrás de ese $63 \%$ que se inclina por emprender acciones que fomenten la igualdad de oportunidades entre mujeres y hombres en la UAA? ¿Qué experiencias asimilan, qué tipo de acciones presentan, qué relaciones de convivencia denuncian? Es algo que debemos leer e intuir con inteligencia para nivelar y atender a sus peticiones explícitas.

Es importante aclarar que en la categoría de personal administrativo se halla incluida una amplia gama de personas: profesionistas, también los hay con posgrados y especializaciones, con estudios y cursos de actualización, gestión y prestación de servicios, docentes de las variadas categorías y niveles; asimismo, con niveles básicos de escolaridad, como es el caso de personas de apoyo, de intendencia, limpieza, vigilancia, jardinería, secretarial, especialmente de aquell@s que ingresaron hace más de 20 años, etcétera. Es una franja de servidores institucionales que representan, utilizan y portan la representatividad de la universidad, dentro y fuera de ella. Ellas y ellos son, visibilizan, identifican, componen y viven en, para y de la universidad. Por otra parte, con cierta frecuencia dan a comprender que las personas de apoyo administrativo son de segunda categoría o de segundo nivel. Su tabulador de ingresos, comparativamente con 1@s docentes, es inferior en grado significativo, tal vez eso mismo sea el origen de esa diferenciación entre ambos segmentos universitarios.

¿Qué proponen para atender tamaña preocupación institucional?

- Realizar una feria con conferencias, pláticas y foros informativos. (31 personas, que representan el 11.5\%).

Resulta interesante leer con detenimiento dichas propuestas, porque están demandando atención, tiempo y preparación para ell@s, ya que muestran preocupación, ansiedad, necesidad y disposición para compartir y aprender de aquellas acciones que se vayan a emprender para cubrir el déficit que apuntan. Esta misma información se ha venido constatando cada vez que se establecen proyectos 
piloto y ofrecimientos de esa naturaleza para el personal de apoyo. En tal sentido, las primeras dos experiencias fueron muy bien acogidas?

- Igualdad de oportunidades. (31, 11.5\%) Es digno de mención aparte que la segunda propuesta también fue avalada por 31 personas, ello denota la preocupación y la urgencia que tiene y significa para ellos ver que la igualdad universitaria sea algo más que discurso, que se manifieste en las condiciones de igualdad que deben reinar en las relaciones laborales y en la comunicación cotidiana. Simple y sencillamente están exigiendo algo elemental: no hacer distingos entre personas, porque en más de una ocasión, las otras respuestas del cuestionario dejan entrever que entre un $6 \%$ y $8 \%$ perciben preferencias o denuncian anomalías en el trato diario en ciertos departamentos de áreas administrativas.

- Capacitación. (21, 7.8\%). Esta tercera recomendación se vincula con la primera, y consiste en la solicitud constante de que se les impartan cursos de capacitación, se les ofrezca variedad de oportunidades de formación, actualización y de cursos para acceder a las convocatorias de promoción de mejores condiciones de trabajo, porque en ello va de por medio un mejor ingreso. Particularmente, han expresado que el tema de equidad de género les interesa y les viene bien, porque es un mecanismo informativo que les permite sentirse valorados, bien acogidos y tomados en cuenta para estos programas particulares. La capacitación es una obligación de las instituciones y empresas hacia sus trabajadores, en el caso de la Universidad, además, es una oportunidad de formar y sumar a los proyectos humanísticos y educativos, de acuerdo a su modelo educativo.

- Tratarnos de igual manera. (19, 7.8\%). Dicha observación es una constante entre trabajadoras y trabajadores porque con

7 Al respecto, debe comentarse que la presentación que se hizo con trabajadores de la Dirección de Infraestructura fue impactante, bien muy recibida, ell@s mostraron plena disposición para las capacitaciones de equidad de género. La segunda experiencia correspondió con la exposición que se tuvo con los integrantes del comité ejecutivo del STUAA. Ambas convivencias se impartieron durante el mes de junio de 2012. 
frecuencia ven justamente la aplicación opuesta, preferencias, mejores condiciones o exclusiones por diversas razones. Por tanto, este llamado es relevante y también urgente; es una petición expresa para enmendar prácticas y ejercicios que denotan desigualdad en la convivencia laboral. Dicha expresión ha de estar presente en las relaciones recíprocas de un@s hacia otr@s, pues somos una comunidad, adicionalmente una sociedad inteligente y con referencias teóricas, culturales y políticas distintas y de mayores alcances que la media de esta sociedad a la que se debe la universidad.

- Compartir actividades. $(6,2.2)$. No menos relevante es crear conciencia de esa empatía o forma de vida que se define como compartir antes que competir o inhibir la participación directa, voluntaria y las iniciativas de las personas deberían ser bienvenidas y festejadas. Compartir es una acción tan profunda y tan compleja, tan exigente y necesaria que denota la esencialidad de una comunidad, máxime cuando hablamos de la universitaria. Todo lo que se haga para atender ésta y otras demandas similares será insuficiente, por ello, tal vez, lo que hace falta es modificar la actitud y las formas de convivencia sutil de toda la comunidad, de modo tal que ese distanciamiento entre la autoridad, 1@s docentes y el personal administrativo es tan exigente, pues también ell@s son trabajadores, personas, seres humanos en situación, que en el modelo educativo vigente suelen recibir un trato "desigual".

- Fomentar la participación equitativa. (3, 1.1\%). Si leemos con mayor curiosidad varias de las sugerencias del personal administrativo, podemos llegar a descubrir que algunas de estas respuestas son afines y convocan la atención hacia objetivos comunes, pero, por ello mismo, nos indican que sí han carencias, falta de comprensión y de empatía y que la persistencia de sus ideas debe valorarse más allá de lo que podría imaginarse; sería preferible que nos pusiéramos en los zapatos o en la situación de1@s otr@s que no somos nosotros, pero que nos convocan para ajustar esas formas de convivencia y de trabajo. Es decir, su dicho de fomentar la participación equitativa o de compartir actividades es un llamado permanente que hay que atender. Lo que más valoramos en una familia o en una comunidad humana es, justamente, la cohesión y 
la confianza que se ejerce y se promueve para que todos sus integrantes crezcan en la misma proporción y con las mismas posibilidades de desempeño. Eso es lo que ha de promover la comunidad universitaria, cada uno desde el espacio que le corresponde. No esperemos órdenes, seamos sensibles y comencemos con la parte que le toca a cada uno. Esa actitud es el comienzo para atender los reclamos administrativos.

NR 148. (54.8\%). No atendieron esta pregunta 148 personas.

Total encuestas 270 .

\section{Personal académico}

¿Qué podemos descubrir a partir del cuestionario aplicado al personal docente?

De las 53 preguntas aplicadas a docentes, a continuación se enlistan únicamente 21 , esto es, las que se conectan con el tema analítico que nos ocupa.

1. ¿Consideras que en la UAA se vive la igualdad de género? $270(81.8 \%)$ sí; 45 (13.6\%) no; no contestó 15 (4.5\%)

2. ¿Hay otras personas en tu área que por ser de diferente sexo ya se les haya otorgado algún ascenso?

3. ¿En el tiempo que tienes en la UAA, te has sentido aparta$\mathrm{da}(\mathrm{O})$ de algún evento por ser mujer/hombre /homosexual?

4. ¿Consideras que haya algún puesto exclusivo para hombres y/o mujeres en la UAA?

$208(63 \%)$ personas dicen que no hay puestos exclusivos para hombres y/o mujeres; 69 (20.9\%) responden que sî y no atendieron la pregunta 53 (16.1\%)

5. ¿Has recibido un trato desigual en las capacitaciones por parte del instructor?

6. Si tu respuesta fue Sí, ¿crees que se debió por el hecho de ser de diferente sexo al del instructor(a)?

7. ¿Crees que el no tener un salario justo se deba por alguna situación de discriminación por diferencia de sexos?

8. ¿Sus compañeros hacen comentarios ofensivos o burlescos hacia mujeres, hombres o a personas homosexuales? 
9. ¿Sus compañeras hacen comentarios ofensivos o burlescos hacia mujeres, hombres o a personas homosexuales?

10. ¿Tus superiores suelen hacer comentarios ofensivos hacia mujeres, hombres o a personas homosexuales?

11. ¿Tus superiores le dan su lugar por igual a los hombres y a las mujeres de su área?

12. ¿La mujer tiene que esforzase más que el hombre para progresar en su trabajo?

13. ¿Le han dicho algún cumplido sobre tu apariencia que te haya incomodado?

14. ¿Ha sido víctima de contacto físico no deseado?

15. ¿Considera que el ambiente que te rodeas es sexista (privilegiar un género por encima del otro)?

16. ¿Le han hecho invitaciones de tipo sexual?

17. ¿Le han chantajeado si no aceptas alguna invitación fuera de la Universidad?

18. ¿Se le ha forzado físicamente a tener contacto de tipo sexual?

19. ¿En términos generales, consideras que existe trato desigual entre hombres y mujeres en la UAA?

20. ¿Crees necesario emprender acciones que fomenten la igualdad de oportunidades entre mujeres y hombres en la UAA?

21. ¿Cuáles acciones consideras más adecuadas para fomentar la igualdad de oportunidades entre hombres y mujeres en la UAA?

Ambientación y contexto

Para comenzar ha de recordarse que las maestras y los maestros representan la base sustancial y fundante más relevante de las instituciones educativas, por lo tanto también de las universidades, como es el caso, porque son los profesionistas, promotores, modeladores y responsables directos de muchas de las acciones que suceden en las aulas, laboratorios y demás espacios y momentos de modelación profesional de las y los alumnos. Pero también porque integrantes del personal docente se hallan presenten en funciones y puestos de responsabilidad de primero y segundo órdenes, asimismo en puestos de dirección central (Rectoría, Direcciones y Centros Académicos), por lo que, aquello que ocurre en la comunidad universitaria, sucede bajo la mirada, la criba, el cuidado y los criterios, éticos, educativos 
y profesionales que estilan y reflejan las y los académicos en su vida universitaria, esto es, su ser humano, profesional y ético se da en el hacer y ser de la universidad.

Regularmente, el tiempo de permanencia de unos y otras transcurre en ese lapso laboral de veinte, treinta o más años de presencia y permanencia activa y eficiente dentro de los espacios, aulas y momentos de una institución superior. Otra consideración más nos lleva a desdoblar la entraña académica, sus habilitaciones y habilidades conseguidas, ya que se han formado y preparado en sus respectivas áreas de conocimiento, habrán destacado en perfiles científicos, tecnológicos y humanísticos particulares y representan un peso y una aportación singular en las distintas responsabilidades que suelen cubrir u ocupar en ese largo trazo de años activos.

Todavía más, la formación cívica, sus valores e ideales, sus sueños y proyectos personales y profesionales se van expresando, activando, sucediendo, aplicando o reteniendo, según sean las oportunidades, de tal modo que, así como en la cabeza de las instituciones superiores, la Rectoría lleva mano y toma las decisiones centrales, hacia el interior y al exterior y se conecta con las otras organizaciones sociales, culturales y políticas de su entorno y de su contexto, también al interior de estas casas de estudios, para el caso de la UAA, en los decanatos, jefaturas, cuerpos académicos, academias y demás espacios de organización, representación y toma de decisiones en la universidad, van dejando sus respectivas huellas, siembran sus anhelos y diseñan una diversidad de programas, proyectos, proyecciones, conceptos teóricos, metodológicos, producciones de investigación y demás productos de docentes. Por ello se aplica a las instituciones superiores en general, y a las universidades en especial, tal concepto, de universitas, como la suma de agentes, organizaciones, personas y demás áreas que las componen y su integración colegiada en conjunto. Esto es, universitas viene a ser la conjunción permanente de personas, proyectos, voluntades, segmentos, escenarios, planes y programas y todo aquello que hay y se conjuga para darle vida, esencia y expresión a la comunidad universitaria.

Lo más sutil y lo más cotidiano que poseemos son aquellas expresiones personales y docentes que identificamos y reconocemos como acciones humanas, actitudes e ideales morales, éticos y personales, y que se reflejan como acciones morales que desgranamos a cada mo- 
mento, en cada oportunidad, en cada hecho que hacemos u omitimos en relación y vinculación con alguna de las personas de nuestra comunidad universitaria. Ese valor intrínseco pocas veces observado es lo que estilamos y dejamos como estela individual de nuestro ser en ese largo recorrido de nuestra vida universitaria. Al término de una y de las vidas profesionales de que hemos sido testigos, lo que más se recuerda y se reconoce son las actitudes y valores morales de los sujetos universitarios.

A continuación se exponen las respuestas dadas por este segmento, en el mismo orden de las dos presentaciones anteriores.

Comentarios a las respuestas

¿Cree necesario emprender acciones que fomenten la igualdad de oportunidades entre mujeres y hombres en la UAA?

El personal académico respondió así: 160 (48.5\%) por el sí, 155 (47\%) por el no y 15 (4.4\%) no respondieron.

Esto es, ellas y ellos dicen que sí hacen falta las acciones correctivas, lo que implica es que tales actividades serán bienvenidas porque promueven y coadyuvan a construir la anhelada igualdad y respeto en la universidad. Si bien la respuesta es muy pareja entre el sí y el no, que $48.5 \%$ recomiende acciones para recrear la igualdad es porque ésta tal vez hace falta en la convivencia diaria, sea en la función docente, de investigación, de apoyo, de coordinación y de solidaridad; de empatías y de tolerancias en la vida común de la universidad. Sí es su respuesta. En cambio $47 \%$ estima que no hace falta, que la igualdad entre la comunidad universitaria es aceptable y que por ahora no sería necesario, menos urgente. ¿Por qué no tomar una iniciativa interesante para apoyar la difusión de acciones que promuevan la igualdad de oportunidades y la disminución de mal trato, exclusión o coacción hacia las personas?

¿Cuáles acciones consideras más adecuadas para fomentar la igualdad de oportunidades entre hombres y mujeres en la UAA?

En particular, para las respuestas emitidas a esta pregunta por 1@s docentes, se hace una excepción, ya que no se glosarán in extenso, como en el caso de estudiantes y personal administrativo, a fin de evitar re- 
peticiones, únicamente nos detenemos en aquellas afirmaciones que reflejan con mayor viveza el carácter y relevancia del personal académico.

Realizar una feria con conferencias, pláticas y foros informativos fue apoyada por 46 docentes, que significa $13.9 \%$; Igualdad de oportunidades recibió 31 referencias, con 9.4\%; Capacitación la decidieron 18 personas, con 5.5\%; Respeto entre compañeros, 16 universitari@s docentes, 4.8\%; Tratarnos de igual manera, con 14 votaciones, el 4.2\%; Concurso trasparente para integrar la junta de Gobierno obtuvo 8 menciones, 2.4\%; Compartir actividades alcanzó 6 registros, $1.8 \%$; Trabajo en equipo lo destacaron dos, $0.6 \%$; Fomentar la participación equitativa, sólo una mención, $0.3 \%$; No respondieron esta pregunta 188, 1 o que representó 57\% de maestros y maestras.

Igualdad de oportunidades. ¿Qué alcance puede tener esta respuesta? Dependerá de la forma de contratación, categoría laboral, tipo de nombramiento, antigüedad, función académica que vive en el momento de la encuesta y de otros ingredientes que no fueron registrados y que se quedan como en el limbo, en la indefinición. Pero apuntarla como una acción útil, conveniente y/o necesaria implica que la desigualdad también se presenta en este segmento universitario y que se convierte en una propuesta de oportunidad para mejorar las relaciones entre ellas y ellos, como sujetos-objetos del diagnóstico y de la universidad, como integrantes especiales del proceso de formación y difusión de los valores de igualdad y respeto en la comunidad universitaria. Puede aventurarse la hipótesis de que a mayor igualdad entre dicho sector, probablemente pueda reflejarse en una convivencia humana más aceptable y respetuosa.

Es importante preguntarnos de qué igualdad están hablando, si de oportunidades laborales, de relaciones humanas, de tolerancia y pluralidad social y académica o de otra idea vinculada con estos aspectos. Asimismo, estas ideas podrían repetirse en las respuestas dadas a respeto entre compañeros y que a trato sea parejo entre iguales.

Concurso trasparente para integrar la Junta de Gobierno. Esta notación recibió ocho menciones, $2.4 \%$. Debe resaltarse que únicamente fue solicitada por las académicas y los académicos, pues, aunque no era el tema del diagnóstico, su mención lleva a señalar que la universidad tiene una tarea pendiente, que, hasta ahora, no ha atendido, y es la posibilidad real de que unas y otros gocen igualdad de oportunidades en la composición de ese órgano colegiado tan importante. Adicionalmente, saliendo un tanto de los propósitos temáticos, esas 
ocho voces piden concurso transparente para su integración. Propiciar la igualdad entre iguales es uno de los propósitos básicos de los estudios de género y de sus tareas a considerar en el corto y mediano plazos del programa institucional de equidad de género.

La última reflexión es para dos respuestas dadas, porque suelen entenderse como afines y presuponen actitudes humanas sencillas o muy complejas, dependiendo del tipo de reflexión que se lleva a cabo. Nos referimos a la propuesta de Compartir actividades y Trabajo en equipo, porque para unos es una tarea irrelevante, innecesaria y sin un alcance o beneficio que valga la pena; en cabio, otros pueden opinar en sentido opuesto. Lo cierto es que las dos acciones son complementarias o paralelas y recomendables para sobrellevar con dignidad las nobles tareas de la universidad. En este sentido habría una virtud humana indispensable: generosidad de la comunidad.

\section{CONSIDERACIONES FINALES}

Primera. Reiterar que la encuesta que estamos comentando es un primer ejercicio que permite acercarnos a conocer las opiniones de la comunidad universitaria sobre un tema tan completo y tan novedoso. ¿Qué es la equidad de género? ¿Qué opina la comunidad?

Segunda. Por ser un primer estudio, tiene algunas deficiencias que poco a poco se irán subsanando mediante la formación y capacitación del equipo coordinador de esta tarea tan humana y tan valiosa, que luego se ampliarán para todas y todos los que conviven en esta universidad.

Tercera. Como se destacó desde el inicio, aquí sólo se tomaron dos de las muchas preguntas que abarcó el diagnóstico, más como una invitación para quienes se interesen por estos temas y deseen aproximarse al Diagnóstico 2011.

Cuarta. Hace falta ofrecer una serie de actividades para difundir el concepto, ideas, análisis, percepciones, alcances e invitaciones para ocuparnos con seriedad de un tema que está vigente en nuestros días, y que busca disminuir los desniveles informativos y prevenir las acciones de violencia en contra de las personas, por razones de género, en nuestra casa de estudios y en nuestra sociedad.

Quinta. Sin lugar a dudas, hay tareas pendientes que debemos atender, algunas con urgencia, otras en el mediano plazo. La educación moral de1@s estudiantes, del personal académico, administrati- 
vo y directivo es una tarea permanente. Desde aquí hay que hacer un llamado para incorporarnos en esta novedosa visión de la equidad de género en la UAA.

Sexta. Conviene cerrar esta reflexión de acercamiento al estudio de género aplicado en la UAA con las dos preguntas que sirvieron de pretexto y de puente para iniciar esta cavilación general.

$¿ C$ Crees necesario emprender acciones que fomenten la igualdad de oportunidades entre mujeres y hombres en la UAA? ¿Menciona qué acciones consideras más adecuadas para fomentar la igualdad de oportunidades entre hombres y mujeres en la UAA? La comunidad universitaria de la Autónoma de Aguascalientes tiene la palabra. 